\title{
AN INEQUALITY FOR FUNCTIONS OF EXPONENTIAL TYPE NOT VANISHING IN A HALF-PLANE
}

\author{
N. K. GOVIL
}

\begin{abstract}
ABSTRACr. Let $f(z)$ be an entire function of order 1, type $\tau$ having no zero in Im $z<0$. If $h_{f}(-\pi / 2)=\tau, h_{f}(\pi / 2)<0$ then it is known that $\sup _{-\infty<x<\infty}\left|f^{\prime}(x)\right|>(\tau / 2) \sup _{-\infty<x<\infty}|f(x)|$. In this paper we consider the case when $f(z)$ has no zero in $\operatorname{Im} z<k, k<0$ and obtain a sharp result.
\end{abstract}

1. If $f(z)$ is an entire function of exponential type $\tau$ and $|f(x)| \leqslant M$ for real $x$, then according to a well-known theorem due to $S$. N. Bernstein $[1, p$. 206]

$$
\left|f^{\prime}(x)\right| \leqslant M \tau, \quad-\infty<x<\infty .
$$

If $h_{f}(\pi / 2)=0$,

$$
h_{f}(\theta)=\limsup _{r \rightarrow \infty} \frac{\log \left|f\left(r e^{i \theta}\right)\right|}{r}
$$

is the indicator function of $f(z)$, and $f(x+i y) \neq 0$ for $y>0$, then it has been proved by Boas [2] that (1.1) can be replaced by

$$
\left|f^{\prime}(x)\right| \leqslant M \tau / 2, \quad-\infty<x<\infty .
$$

This result of Boas is in fact a generalization of the Erdös conjecture proved by Lax [4] because the class of asymmetric entire functions of exponential type $\tau$ includes all functions $p\left(e^{i z}\right)$ where $p(z)$ is a polynomial of degree $n \leqslant[\tau]$ and $p(z) \neq 0$ in $|z|<1$.

For polynomials having all their zeros in $|z| \leqslant 1$, we have the following result due to Turán [6].

THEOREM A. If $p(z)$ is a polynomial of degree $n$ having all its zeros in $|z| \leqslant 1$, then

$$
\max _{|z|=1}\left|p^{\prime}(z)\right| \geqslant \frac{n}{2} \max _{|z|=1}|p(z)| \text {. }
$$

As a generalization of Theorem $A$, an inequality analogous to (1.3) for entire functions of order 1, and type $\tau$ has been obtained by Rahman [5] and for polynomials having all its zeros in $|z| \leqslant K, K \geqslant 0$ by Govil [3]. In this

Received by the editors May 24, 1974.

AMS (MOS) subject classifications (1970). Primary 30A64; Secondary 30A04, 30A40.

Key words and phrases. Special classes of entire functions and growth estimates, inequalities in the complex domain, extremal problems.

(c) American Mathematical Society 1977 
paper we generalize the result due to Rahman [5] and due to Govil [3] (in the case $K \geqslant 1$ ) and prove the following.

THEOREM. Let $f(z)$ be an entire function of order 1 , type $\tau$ having all its zeros in $\operatorname{Im} z \geqslant k, k \leqslant 0$. If $h_{f}(\pi / 2) \leqslant 0, h_{f}(-\pi / 2)=\tau$ then

$$
\sup _{-\infty<x<\infty}\left|f^{\prime}(x)\right| \geqslant \frac{\tau}{1+\exp (\tau|k|)} \sup _{-\infty<x<\infty}|f(x)| .
$$

The result is best possible with equality for the function

$$
f(z)=\left(\frac{e^{i \tau z}-e^{-\tau k}}{1+e^{-\tau k}}\right) .
$$

2. For the proof of the theorem, we need the following lemmas.

LEMMA 1. If $f(z)$ is an entire function of exponential type $\tau$ and $|f(x)| \leqslant M$, $-\infty<x<\infty$, then

$$
|f(x+i y)| \leqslant M e^{\tau|y|}, \quad-\infty<x<\infty,-\infty<y<\infty .
$$

Lemma 1 is a simple consequence of the Phragmén-Lindelöf principle and follows immediately from a result due to Pólya and Szegö (see $[1$, p. 82, Theorem 6.2.4]).

LEMMA 2. Let $f(z)$ be an entire function of order 1 , type $\tau, h_{f}(\pi / 2) \leqslant 0$, $|f(x)| \leqslant M,-\infty<x<\infty$, and let $g(z)=e^{i \tau z} \operatorname{con}\{f(\bar{z})\}$, where $\operatorname{con}\{f(\bar{z})\}$ denotes the conjugate of $f(\bar{z})$. Then type $g \leqslant \tau$.

Proof of Lemma 2. If $g(z)=e^{i \tau z} \operatorname{con}\{f(\bar{z})\}$ is an entire function of order less than 1 , then obviously type $g \leqslant \tau$, hence it is sufficient to prove the result when $g(z)$ is of order 1 .

If $z=r e^{i \theta}$ is a point of the upper half-plane, then

$$
\left|g\left(r e^{i \theta}\right)\right|=e^{-\tau r \sin \theta}\left|f\left(r e^{-i \theta}\right)\right|,
$$

which gives by Lemma 1 ,

$$
\begin{aligned}
\left|g\left(r e^{i \theta}\right)\right| & \leqslant e^{-\tau r \sin \theta} e^{\tau r \sin \theta} \sup _{-\infty<x<\infty}|f(x)| \\
& =\sup _{-\infty<x<\infty}|f(x)| .
\end{aligned}
$$

If $z=r e^{i \theta}$ lies in the lower half-plane, the point $z=r e^{-i \theta}$ will lie in the upper half-plane and since $h_{f}(\pi / 2) \leqslant 0$, hence it follows by a result due to Pólya and Szegö (see [1, p. 82, Theorem 6.2.4]) that

$$
\begin{aligned}
\left|g\left(r e^{i \theta}\right)\right| & =e^{-\tau r \sin \theta}\left|f\left(r e^{-i \theta}\right)\right| \\
& \leqslant e^{-\tau r \sin \theta} \sup _{-\infty<x<\infty}|f(x)| \\
& \leqslant e^{\tau r} \sup _{-\infty<x<\infty}|f(x)| .
\end{aligned}
$$

On combining (2.2) and (2.3), we get 


$$
\left|g\left(r e^{i \theta}\right)\right| \leqslant e^{\tau r} \sup _{-\infty<x<\infty}|f(x)|, \quad 0 \leqslant \theta<2 \pi,
$$

which gives that type $g \leqslant \tau=\operatorname{type} f$, and Lemma 2 follows.

LEMMA 3. If $f(z)$ is an entire function of order 1 , type $\tau$ such that $h_{f}(-\pi / 2)$ $=\tau, h_{f}(\pi / 2) \leqslant 0, f(z)$ has all its zeros in $\operatorname{Im} z \geqslant k, k \leqslant 0$, then

$$
\sup _{-\infty<x<\infty}\left|g^{\prime}(x)\right| \leqslant e^{\tau|k|} \sup _{-\infty<x<\infty}\left|f^{\prime}(x)\right|,
$$

where as in Lemma $2, g(z)$ stands for $e^{i \tau z} \operatorname{con}\{f(\bar{z})\}$ and $\operatorname{con}\{f(\bar{z})\}$ for the conjugate of $f(\bar{z})$.

Proof of Lemma 3. Let $F(z)=f(z+i k)$ and $G(z)=e^{i \tau z} \operatorname{con}\{F(\bar{z})\}=$ $e^{-\tau k} g(z-i k)$, where $\operatorname{con}\{F(\bar{z})\}$ denotes the conjugate of $\{F(\bar{z})\}$. Since $f(z)$ has all its zeros in $\operatorname{Im} z \geqslant k, k \leqslant 0, h_{f}(-\pi / 2)=\tau, h_{f}(\pi / 2) \leqslant 0$, the function $F(z)$ is an entire function of order 1 , type $\tau$, has no zero in $\operatorname{Im} z<0$, $h_{F}(-\pi / 2)=\tau$ and $h_{F}(\pi / 2) \leqslant 0$. Therefore the function $F(z)$ belongs to the class $P$. Further $F_{1}(z)=e^{i \tau z / 2} \operatorname{con}\{F(\bar{z})\}$ is an entire function of exponential type having no zero in $\operatorname{Im} z>0$ and satisfying $h_{F_{1}}(\pi / 2) \geqslant h_{F_{1}}(-\pi / 2)$. Hence applying a result due to Levin (see [1, p. 129, Theorem 7.8.1]) to the function $F_{1}(z)$, we get

$$
\left|e^{i \tau z / 2} \operatorname{con}\{F(\bar{z})\}\right| \geqslant\left|e^{i \tau \bar{z} / 2} \operatorname{con}\{F(z)\}\right| \text { for } \operatorname{Im} z \geqslant 0,
$$

which implies

$$
\left|e^{i \tau \bar{z} / 2} \operatorname{con}\{F(z)\}\right| \geqslant\left|e^{i \tau z / 2} \operatorname{con}\{F(\bar{z})\}\right| \text { for } \operatorname{Im} z \leqslant 0,
$$

and which implies

$$
\left|F(z) e^{-i \tau z / 2}\right| \geqslant\left|e^{i \tau z / 2} \operatorname{con}\{F(\bar{z})\}\right| \text { for } \operatorname{Im} z<0 .
$$

Thus

$$
|F(z)| \geqslant\left|e^{i \tau z} \operatorname{con}\{F(\bar{z})\}\right| \text { for } \operatorname{Im} z \leqslant 0 .
$$

Since $G(z)=e^{i \tau z} \operatorname{con}\{F(\bar{z})\}$, we get

$$
|F(z)| \geqslant|G(z)| \text { for } \operatorname{Im} z \leqslant 0 .
$$

For $k \leqslant 0$, let $F_{k}(z)$ denote the function $F(z+i k)$ and $G_{k}(z)$ the function $G(z+i k)$. Then the function $F_{k}(z)$ is an entire function of order 1 , type $\tau$. Also by Lemma 2, the function $G_{k}(z)$ is an entire function of exponential type $\leqslant \tau$. Since $F(z)$ has no zero in $\operatorname{Im} z<0$, therefore $F_{k}(z)$ has no zero in $\operatorname{Im} z<-k$, and hence no zero in $\operatorname{Im} z<0$, because $k \leqslant 0$. Further because $h_{F_{k}}(-\pi / 2)=h_{F}(-\pi / 2)=\tau, h_{F_{k}}(\pi / 2)=h_{F}(\pi / 2) \leqslant 0$, we get $h_{F_{k}}(-\pi / 2) \geqslant$ $h_{F_{k}}(\pi / 2)$ and therefore $F_{k}(z)$ belongs to the class $P$. Thus $G_{k}(z)$ is an entire function of exponential type $\leqslant \tau$ and $F_{k}(z)$ an entire function of class $P$, order 1 and type $\tau$. Also by (2.5) we have $\left|G_{k}(x)\right| \leqslant\left|F_{k}(x)\right|,-\infty<x<\infty$, hence applying a result due to Levin (see [1, p. 226, Theorem 11.7.2]) and the fact that differentiation is a $B$-operator, we get $\left|G_{k}^{\prime}(x)\right| \leqslant\left|F_{k}^{\prime}(x)\right|,-\infty<x$ $<\infty$, which implies 


$$
\left|G^{\prime}(x+i k)\right| \leqslant\left|F^{\prime}(x+i k)\right|, \quad-\infty<x<\infty, k \leqslant 0 .
$$

Since $\left|F^{\prime}(x+i k)\right|=\left|f^{\prime}(x+2 i k)\right|$, and $\left|G^{\prime}(x+i k)\right|=e^{-\tau k}\left|g^{\prime}(x)\right|$, (2.6) gives,

$$
\left|g^{\prime}(x)\right| \leqslant e^{\tau k}\left|f^{\prime}(x+2 i k)\right|, \quad-\infty<x<\infty .
$$

Lastly applying the inequality (2.1) to $\left|f^{\prime}(x+2 i k)\right|$ and combining it with (2.7) we get

$$
\left|g^{\prime}(x)\right| \leqslant e^{\tau|k|} \sup _{-\infty<x<\infty}\left|f^{\prime}(x)\right|,
$$

from which the lemma follows.

LEMMA 4. If $f(z)$ is an entire function of exponential type $\tau$, then

$$
\sup _{-\infty<x<\infty}\left|f^{\prime}(x)\right|+\sup _{-\infty<x<\infty}\left|g^{\prime}(x)\right| \geqslant \tau \sup _{-\infty<x<\infty}|f(x)|,
$$

where $g(z)$ is the same as defined in Lemma 3.

Proof of Lemma 4. From the definition of $g(z)$ it follows that on real axes.

$$
\begin{aligned}
\left|g^{\prime}(x)\right| & =\left|e^{i \tau x} \overline{f^{\prime}(x)}+i \tau e^{i \tau x} \overline{f(x)}\right| \\
& \geqslant \tau|f(x)|-\left|f^{\prime}(x)\right| .
\end{aligned}
$$

Thus for $-\infty<x<\infty,\left|f^{\prime}(x)\right|+\left|g^{\prime}(x)\right| \geqslant \tau|f(x)|$, which gives

$$
\sup _{-\infty<x<\infty}\left|f^{\prime}(x)\right|+\sup _{-\infty<x<\infty}\left|g^{\prime}(x)\right| \geqslant \tau \sup _{-\infty<x<\infty}|f(x)|,
$$

and Lemma 4 is proved.

3. Proof of the Theorem. We have by Lemma 3,

$$
\sup _{-\infty<x<\infty}\left|g^{\prime}(x)\right| \leqslant e^{\tau|k|} \sup _{-\infty<x<\infty}\left|f^{\prime}(x)\right| .
$$

Also by Lemma 4,

$$
\sup _{-\infty<x<\infty}\left|f^{\prime}(x)\right|+\sup _{-\infty<x<\infty}\left|g^{\prime}(x)\right| \geqslant \tau \sup _{-\infty<x<\infty}|f(x)| .
$$

Combining (3.1) and (3.2) we get

$$
\sup _{-\infty<x<\infty}\left|f^{\prime}(x)\right|+e^{\tau|k|} \sup _{-\infty<x<\infty}\left|f^{\prime}(x)\right| \geqslant \tau \sup _{-\infty<x<\infty}|f(x)|,
$$

which implies

$$
\sup _{-\infty<x<\infty}\left|f^{\prime}(x)\right| \geqslant \frac{\tau}{1+e^{\tau|k|}} \sup _{-\infty<x<\infty}|f(x)| .
$$

This completes the proof of the theorem.

I am extremely grateful to Professor Q. I. Rahman for his kind help in the preparation of this paper.

\section{REFERENCES}

1. R. P. Boas, Jr., Entire functions, Academic Press, New York, 1954.

2. , Inequalities for asymmetric entire functions, Illinois J. Math. 1 (1957), 94-97. 
3. N. K. Govil, On the derivative of a polynomial, Proc. Amer. Math. Soc. 41 (1973), 543-546.

4. P. D. Lax, Proof of a conjecture of P. Erdös on the derivative of a polynomial, Bull. Amer. Math. Soc. 50 (1944), 509-513.

5. Q. I. Rahman, On asymmetric entire functions, Proc. Amer. Math. Soc. 14 (1963), 507-508.

6. P. Turán, Über die Ableitung von polynomen, Compositio Math. 7 (1939), 89-95.

Department of Mathematics, Indian Institute of Technology, New Delhi-110029, India

Département de Mathématiques, Université de Montréal, Montréal, Canada 